This item was submitted to Loughborough's Research Repository by the author.

Items in Figshare are protected by copyright, with all rights reserved, unless otherwise indicated.

\title{
A hybrid phase-based single frequency estimator
}

PLEASE CITE THE PUBLISHED VERSION

PUBLISHER

(C) IEEE

VERSION

VoR (Version of Record)

LICENCE

CC BY-NC-ND 4.0

\section{REPOSITORY RECORD}

Zhuo, Zhang, Andreas Jakobsson, M.D. Macleod, and Jonathon Chambers. 2019. "A Hybrid Phase-based Single Frequency Estimator". figshare. https://hdl.handle.net/2134/5772. 
This item was submitted to Loughborough's Institutional Repository (https://dspace.lboro.ac.uk/) by the author and is made available under the following Creative Commons Licence conditions.

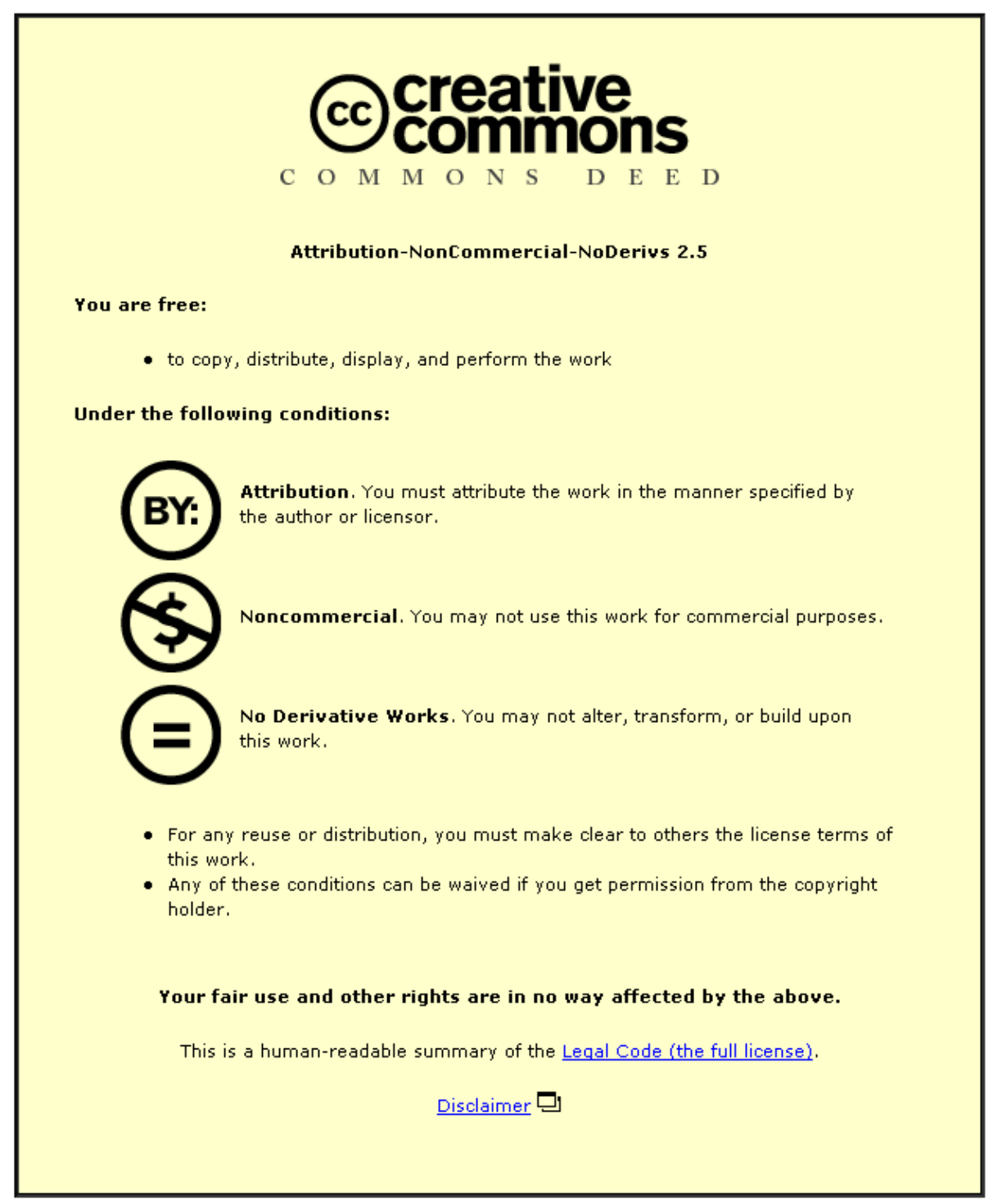

For the full text of this licence, please go to: http://creativecommons.org/licenses/by-nc-nd/2.5/ 


\title{
A Hybrid Phase-Based Single Frequency Estimator
}

\author{
Zhuo Zhang, Student Member, IEEE, Andreas Jakobsson, Member, IEEE, Malcolm D. Macleod, Member, IEEE, and \\ Jonathon A. Chambers, Senior Member, IEEE
}

\begin{abstract}
The topic of low computational complexity frequency estimation of a single complex sinusoid corrupted by additive white Gaussian noise has received significant attention over the last decades due to the wide applicability of such estimators in a variety of fields. In this letter, we propose a computationally fast and statistically improved hybrid phase-based estimator that outperforms other recently proposed approaches, lowering the signal-to-noise ratio at which the Cramér-Rao lower bound is closely followed.
\end{abstract}

Index Terms-Classification theory and methods, computation, detection, estimation, fast algorithms, transforms.

\section{INTRODUCTION}

$\mathbf{I}$ $\mathrm{N}$ a wide variety of application areas, such as biomedicine, communications, and radar, one often encounters a need to find a low computational complexity estimate of the dominant frequency component of data that are assumed to consist of a single complex sinusoid corrupted by additive white Gaussian noise, and the topic has, as a result, attracted significant interest over the last decades (see, e.g., [1]-[14] and the references therein). The problem can be briefly stated as follows; consider the data sequence

$$
y(t)=\beta e^{i(\omega t+\theta)}+n(t)
$$

where $\beta \in \mathbb{R}, \omega$, and $\theta \in[0,2 \pi)$ denote the deterministic but unknown amplitude, frequency, and initial phase, respectively, of a complex sinusoid. Further, $n(t)$ is a circular zero-mean complex Gaussian white noise with variance $\sigma_{n}^{2}$. Then, given the sequence $y(t)$, for $t=0, \ldots, N-1$, the problem is simply to estimate $\omega$ with a low computational complexity and statistically efficient estimator. In [2], Rife and Boorstyn derived the maximum likelihood (ML) estimator of $\omega$ and proposed a statistically efficient approximative ML approach involving both a combined coarse and fine search using the fast Fourier transform (FFT) algorithm. However, zeropadding is often required to obtain sufficient resolution, requiring $\mathcal{O}\left(N^{\prime} \log _{2} N^{\prime}\right)$ operations, where $N^{\prime}$ is the size of the desired frequency grid, with typically $N^{\prime} \gg N$. Further, an iterative linear prediction (ILP) approach requiring $\mathcal{O}\left(N \log _{2} N\right)$ operations was suggested in [15], showing similar performance to that of the

Manuscript received February 8, 2005; revised April 14, 2005. The associate editor coordinating the review of this manuscript and approving it for publication was Dr. Israel Cohen.

Z. Zhang and J. A. Chambers are with the Centre of Digital Signal Processing, Cardiff School of Engineering, Cardiff University, Cardiff CF24 OYF, U.K. (e-mail: zhuo.zhang@ @ieee.org; ChambersJ@cardiff.ac.u).

A. Jakobsson is with the Department of Electrical Engineering, Karlstad University, SE-651 88 Karlstad, Sweden (e-mail: andreas.jakobsson@ ieee.org).

M. D. Macleod is with QinetiQ Ltd, St. Malvern WR14 3PL, U.K. (e-mail: m.macleod@ signal.qinetiq.com).

Digital Object Identifier 10.1109/LSP.2005.853043
ML. A variety of phase-based methods requiring only $\mathcal{O}(N)$ operations have been developed. In [3], for example, Tretter proposed a phase-based approach simplifying the problem to a linear regression on the phase. The method is based on a phase unwrapping algorithm, requiring a very high signal-to-noise ratio (SNR) (SNR 》1), here defined as $\mathrm{SNR}=\beta^{2} / \sigma_{n}^{2}$, to work well. Later, Kay proposed a modified version of Tretter's algorithm avoiding the use of the phase unwrapping algorithm [4]. The method, here termed Kay's weighted phase average (KWPA) estimator, can be shown to attain the Cramér-Rao lower bound (CRLB) for sufficiently high SNR, but the method is in general biased, and the SNR for which the CRLB is achieved depends on the underlying frequency [6], [8], [12]. As a result, the focus of recent contributions has mainly been aimed to reduce the SNR threshold [10], the frequency dependency of the threshold [7], or both [11], [14]. In this letter, we propose a hybrid method combining the ideas in [10], [11], and [14] to show the performance close to that of ML or ILP but only requiring $\mathcal{O}(N)$ operations. The hybrid estimate is based on an initial coarse estimate of the unknown frequency using the uniformly weighted linear predictor (UWLP) method [1], [4]; this estimate is used to remove the frequency dependence of the SNR threshold. This SNR threshold is then further reduced via a combination of using an averaging filter, as suggested in [10], and an outlier removal scheme, as proposed in [14]. Finally, a refined frequency estimate is formed along the lines proposed in [10] and [11].

The remainder of this letter is organized as follows: In the following section, the proposed hybrid method is introduced, including a discussion of the different involved algorithms. Then, Section III contains numerical simulations illustrating the performance of the hybrid estimator. Finally, Section IV contains our conclusions.

\section{Hybrid Phase-BASEd Frequency Estimator}

As suggested in [3], the data model in (1) can be written as

$$
y(t)=[1+v(t)] \beta e^{i(\omega t+\theta)}
$$

where $v(t)=\beta^{-1} n(t) e^{-i(\omega t+\theta)}$ is a complex white sequence. Let $v_{r}(t)$ and $v_{i}(t)$ denote the real and the imaginary parts of $v(t)$, respectively. Then, for high SNR

$$
1+v(t) \approx e^{i \arctan v_{i}(t)} \approx e^{i v_{i}(t)}
$$

allowing the approximation

$$
y(t) \approx \beta e^{i \phi(t)}
$$

where $\phi(t)=\omega t+\theta+v_{i}(t)$. Most of the recent phase-based approaches exploit this approximation, allowing the phase to 
be approximately estimated from the difference of the adjacent phase values, i.e.,

$$
\Delta \phi(t) \triangleq \arg \left[y^{*}(t) y(t+1)\right] \approx \omega+v_{i}(t+1)-v_{i}(t)
$$

where $(\cdot)^{*}$ denotes the complex conjugate, suggesting the UWLP frequency estimator [1]

$$
\hat{\omega}_{c}=\arg \left[\frac{1}{N-1} \sum_{t=0}^{N-2} y^{*}(t) y(t+1)\right] .
$$

The UWLP estimator is unbiased but statistically inefficient with variance [4], [8]

$$
\operatorname{var}\left(\hat{\omega}_{c}\right)=\frac{1}{(N-1)^{2} \mathrm{SNR}}
$$

As suggested in [7] and [14], we will here use the UWLP estimate to form a downshifted signal $y_{d}(t)$ to remove the frequency dependence of the SNR threshold, i.e.,

$$
y_{d}(t)=y(t) e^{-i \hat{\omega}_{c} t} .
$$

To further reduce the SNR threshold, Kim et al. proposed averaging the downshifted signal using a simple $K$-tap averaging filter, forming [10]

$$
y_{f}(t)=\frac{1}{K} \sum_{k=0}^{K-1} y_{d}(t+k) .
$$

Such an averaging can be shown to lower the SNR threshold up to $10 \log _{10} K \mathrm{~dB}$ but can only be applied to the downshifted signal as it restricts the frequency range to $(-\pi / K, \pi / K]$. Similar to (5), the adjacent phase difference of (9) can be formed as

$$
\Delta \phi_{f}(t)=\arg \left[y_{f}^{*}(t) y_{f}(t+1)\right]=\omega_{f}+u_{c}(t)
$$

where $u_{c}(t)$ is given by (23) for a general $K$. It is worth noting that the noise process $u_{c}(t)$ will now be colored due to the average filtering [13].

As shown in [5], the SNR threshold behavior of the phasebased frequency estimators is affected by cumulative $\pm 2 \pi$ phase errors resulting from the effect of the additive noise. This effect can be countered for by introducing an outlier detection scheme. Recently, an effective scheme was proposed in [14], where $\pm 2 \pi$ outliers are detected if $\left|\beta_{t}\right|>\left|\beta_{t-1}\right|,\left|\beta_{t}\right|>\left|\beta_{t+1}\right|$, and $\left|\beta_{t}\right|>$ $\lambda$, with

$$
\beta_{t}=\Delta \phi_{f}(t-1)+\Delta \phi_{f}(t)+\Delta \phi_{f}(t+1)
$$

where $\beta_{-1}=\beta_{N-K}=0$ and $\Delta \phi_{f}(-1)=\Delta \phi_{f}(N-K)=0$. Thus, the outliers can be removed as follows:

$$
\Delta \tilde{\phi}_{f}(t)= \begin{cases}\Delta \phi_{f}(t)-\operatorname{sign}\left(\beta_{t}\right) 2 \pi, & \text { if outlier detected } \\ \Delta \phi_{f}(t), & \text { otherwise }\end{cases}
$$

for $t=0, \ldots, N-K-1$. Here, $\lambda$ is a user parameter selected to minimize the mean-squared error (MSE). We note that for large $\lambda$, some outliers are not detected, increasing the MSE. Similarly, for small $\lambda$, some events that are not outliers are wrongly detected as outliers, causing errors and increasing the MSE. Therefore, there is an optimum value of $\lambda$ that can be determined empirically. We remark that, according to our experience, the performance of the resulting estimator is only slightly affected by varying $\lambda$ [16]. After the SNR threshold reduction using (10) and (12), further improvement can be achieved by taking into account the coloration of the noise term in (10). This can be achieved using the suggested four-channel filter bank (FCFB) method $^{1}$ in [11] and [13], whereby the frequency correction term $\hat{\omega}_{f}$ can be found as

$$
\hat{\omega}_{f}=\sum_{t=1}^{(N-K) / K} q(t) \sum_{m=1}^{K-1} \Delta \tilde{\phi}_{f}(t K-m)
$$

where

$$
q(t)=\frac{6 t K(N-t K)}{N^{3}-N K^{2}}
$$

with $t=1,2, \ldots,(N-K) / K$. Combined with the coarse estimate, the hybrid frequency estimate is found as

$$
\hat{\omega}_{h}=\hat{\omega}_{c}+\hat{\omega}_{f} .
$$

In summary, the proposed hybrid estimator is found by initially forming the downshifted signal in (8) using the UWLP estimate in (6). Then, the phase difference of the filtered signal is formed using (10), followed by the outlier removal scheme in (12). Finally, the refined frequency estimate is formed as (15), using (13). It is worth stressing that the hybrid method differs from previously suggested approaches in that it combines all the above steps; the FCFB method does not include the outlier removal scheme in (12). Similarly, the method proposed in [14], hereafter termed the outlier removal estimator (ORE), does not include the filtering in (10).

\section{SimULATIONS}

In this section, we will briefly examine the performance of the proposed estimator. Initially, we consider $N=24$ data samples containing a single complex sinusoid with frequency $\omega=0.75 \pi$ and examine the estimated MSE as a function of the SNR. Fig. 1 illustrates the MSE for the proposed hybrid estimator, using a $K=6$ tap averaging filter and outlier detection threshold $\lambda=4$, as compared to the UWLP approach [1], the FCFB approach following [11], the ORE approach [14], the ILP approach using three iterations [15], and the corresponding CRLB as given in [2]. As is clear from the figure, the hybrid estimator shows similar but slightly better performance than the ILP estimator; however, the latter requires $\mathcal{O}\left(N \log _{2} N\right)$ operations, whereas the former only requires $\mathcal{O}(N)$ operations. We note that the hybrid estimator is statistically improved, closely following the CRLB at a lower SNR threshold than the other examined $\mathcal{O}(N)$ methods. Further, the hybrid method uniformly yields a lower MSE than the other methods. It is worth noting that the

${ }^{1}$ It is worth noting that the FCFB applies a different set of weights than those used in the KWPA approach. 


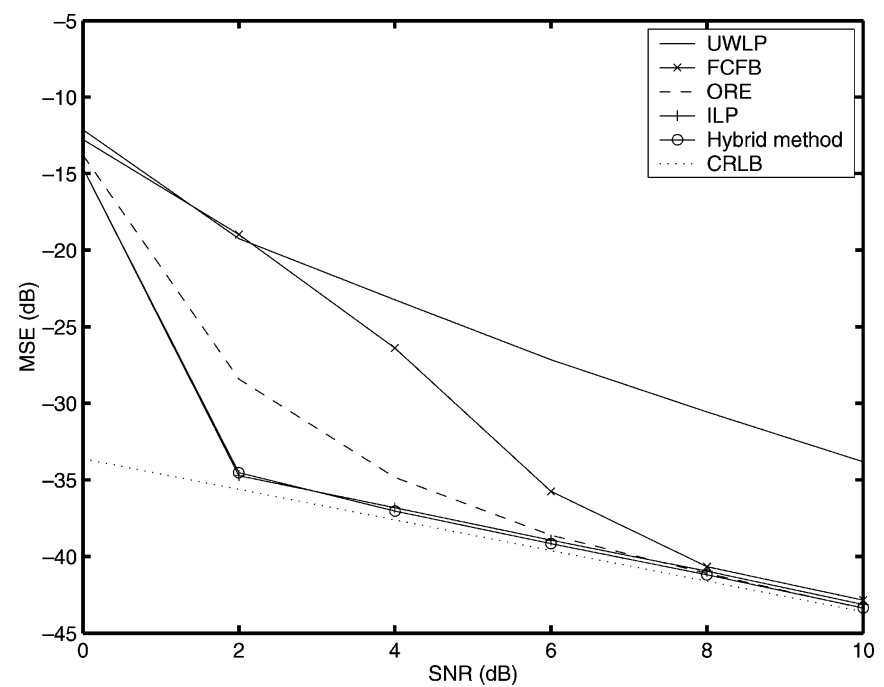

Fig. 1. MSE of the examined estimators as a function of the SNR.

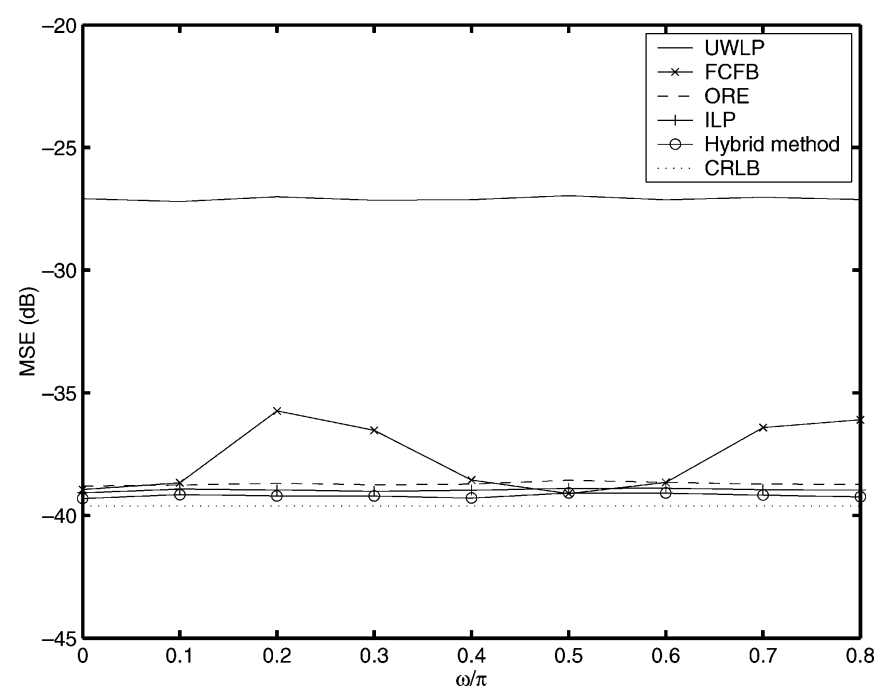

Fig. 2. MSE of the examined estimators as a function of the underlying frequency, for $\mathrm{SNR}=6 \mathrm{~dB}$.

hybrid estimator will suffer some performance degradation due to the introduced averaging in (9), as pointed out in [10]. This explains why the MSE of the proposed hybrid estimator cannot exactly reach the CRLB, as shown in Fig. 1 and in other figures. As is well known, the performance of single frequency estimators is often affected by the underlying frequency. Figs. 2 and 3 illustrate how the MSE varies as a function of the frequency of the sinusoid $\omega$ for SNR $=6 \mathrm{~dB}$ and $\mathrm{SNR}=4 \mathrm{~dB}$, respectively. As seen in the figures, the hybrid estimator is uniformly achieving a lower MSE than the other approaches and is essentially independent of the true frequency. Further, it is clear that the FCFB approach is significantly affected by the frequency, whereas the ORE approach is showing a similar robustness as the hybrid approach, although with a somewhat worse performance. Here, all the simulation results have been obtained using $10^{4}$ Monte Carlo simulations.

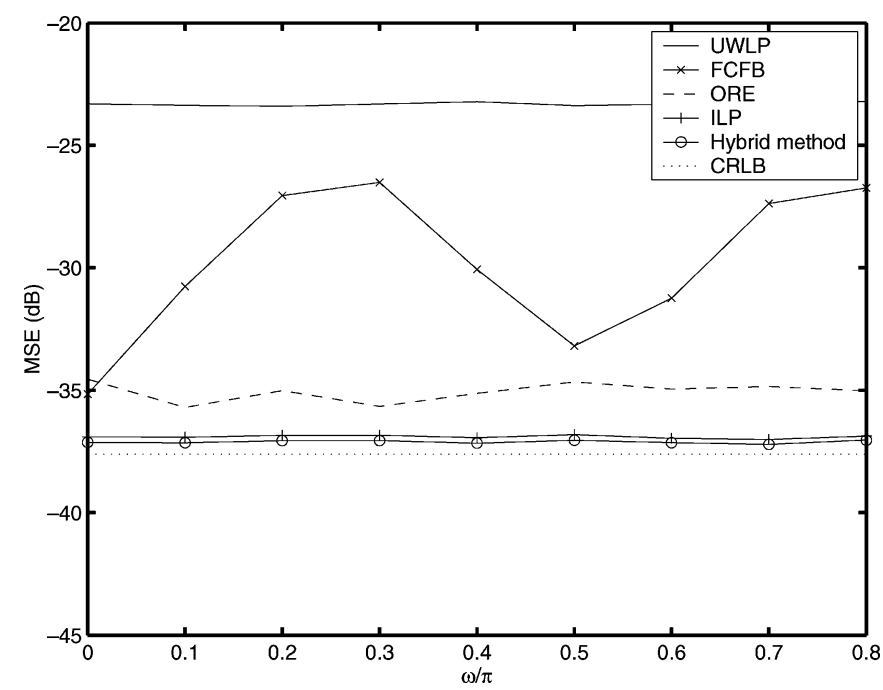

Fig. 3. MSE of the examined estimators as a function of the underlying frequency, for $\mathrm{SNR}=4 \mathrm{~dB}$.

\section{CONCLUSION}

In this letter, we have proposed a low computational complexity hybrid phase-based single frequency estimator combining previously proposed SNR threshold reduction approaches with a recent outlier removal scheme. Numerical simulations indicate that the proposed estimator achieves a lower MSE than other available techniques, closely following the CRLB at a lower SNR threshold. Furthermore, in contrast to many other techniques, the hybrid estimator's performance is found to be essentially independent of the true frequency.

\section{APPENDIX}

In this Appendix, we derive an expression for the noise process $u_{c}(t)$, given in (10), for a general $K$. Let $\omega_{f}=\omega-\hat{\omega}_{c}$. Then, using (2), $y_{f}(t)$ can be expressed as

$$
y_{f}(t)=\frac{\beta}{K} e^{i \omega_{f} t+i \theta} \sum_{k=0}^{K-1}[1+v(t+k)] e^{i \omega_{f} k}
$$

for $t=0, \ldots, N-K$. Introduce

$$
\begin{gathered}
\Psi_{K} \triangleq \sum_{k=0}^{K-1} e^{i(k K-1) \omega_{f} / K} \\
\Phi_{v}^{K}(t) \triangleq \sum_{k=0}^{K-1} v(t+k) e^{i(k K-1) \omega_{f} / K} .
\end{gathered}
$$

Then

$$
\begin{aligned}
y_{f}(t) & =\frac{\beta}{K} e^{i \omega_{f} t+i \theta+i \omega_{f} / K}\left[\Psi_{K}+\Phi_{v}^{K}(t)\right] \\
& =\frac{\beta \Psi_{K}}{K} e^{i \omega_{f} t+i \theta+i \omega_{f} / K}\left[1+\Psi_{K}^{-1} \Phi_{v}^{K}(t)\right] .
\end{aligned}
$$

Thus, the argument of $y_{f}(t)$ can be expressed as

$$
\arg \left[y_{f}(t)\right]=\arg \left[\Psi_{K}\right]+\omega_{f} t+\theta+\frac{\omega_{f}}{K}+\arg \left[1+\Psi_{K}^{-1} \Phi_{v}^{K}(t)\right]
$$


implying that the phase difference from adjacent samples $\Delta \phi_{f}(t)$ can be expressed as

$$
\Delta \phi_{f}(t)=\omega_{f}+u_{c}(t)
$$

where for $t=0, \ldots, N-K-1$

$$
u_{c}(t) \triangleq \arg \left[1+\Psi_{K}^{-1} \Phi_{v}^{K}(t+1)\right]-\arg \left[1+\Psi_{K}^{-1} \Phi_{v}^{K}(t)\right] .
$$

Then, using the approximation in (3), (20) can, for high SNR, be approximated as

$$
u_{c}(t) \approx \operatorname{Im}\left\{\Psi_{K}^{-1}\left[\Phi_{v}^{K}(t+1)-\Phi_{v}^{K}(t)\right]\right\}
$$

where $\operatorname{Im}\{x\}$ denotes the imaginary part of $x$. We note that using a first-order Taylor expansion

$$
\Phi_{v}^{K}(t) \approx \sum_{k=0}^{K-1} v(t+k)
$$

as $\omega_{f}$ is small due to the downshifting, implying that

$$
u_{c}(t) \approx \operatorname{Im}\left\{\Psi_{K}^{-1}[v(t+K)-v(t)]\right\} .
$$

We note that for $K=2$, (23) yields the expression given in [10], i.e.,

$$
u_{c}(t) \approx \frac{v_{i}(t+2)-v_{i}(t)}{2 \cos \left(\frac{\omega_{f}}{2}\right)}
$$

\section{REFERENCES}

[1] G. W. Lank, I. S. Reed, and G. E. Pollon, "A semicoherent detection and Doppler estimation statistic," IEEE Trans. Aerosp. Electron. Syst., vol. AES-9, no. 2, pp. 151-165, Mar. 1973.
[2] D. C. Rife and R. R. Boorstyn, "Single-tone parameter estimation from discrete-time observations," IEEE Trans. Inf. Theory, vol. IT-20, no. 5, pp. 591-598, Sep. 1974.

[3] S. A. Tretter, "Estimating the frequency of a noisy sinusoid by linear regression," IEEE Trans. Inf. Theory, vol. IT-31, no. 6, pp. 832-835, Nov. 1985.

[4] S. Kay, "A fast and accurate single frequency estimator," IEEE Trans. Acoust., Speech, Signal Process., vol. 37, no. 12, pp. 1987-1990, Dec. 1989.

[5] S. W. Lang and B. R. Musicus, "Frequency estimation from phase differences," in Proc. ICASSP, Glasgow, U.K., 1989, pp. 2140-2143.

[6] B. C. Lovell and R. C. Williamson, "The statistical performance of some instantaneous frequency estimators," IEEE Trans. Signal Process., vol. 40, no. 7, pp. 1708-1723, Jul. 1992.

[7] V. Clarkson, "Efficient single frequency estimators," in Int. Symp. Signal Process. Appl., Gold Coast, Australia, 1992, pp. 327-330.

[8] V. Clarkson, P. J. Kootsookos, and B. G. Quinn, "Analysis of the variance threshold of Kay's weighted linear predictor frequency estimator," IEEE Trans. Signal Process., vol. 42, no. 9, pp. 2370-2379, Sep. 1994.

[9] P. Händel, "On the performance of the weighted linear predictor frequency estimator," IEEE Trans. Signal Process., vol. 43, no. 12, pp. 3070-3071, Dec. 1995.

[10] D. Kim, M. J. Narasimha, and D. C. Cox, "An improved single frequency estimator,” IEEE Signal Process. Lett., vol. 3, no. 7, pp. 212-214, Jul. 1996.

[11] M. L. Fowler and J. A. Johnson, "Extending the threshold and frequency range for phase-based frequency estimation," IEEE Trans. Signal Process., vol. 47, no. 10, pp. 2857-2863, Oct. 1999.

[12] B. G. Quinn, “On Kay's frequency estimator," J. Time Series Anal., vol. 21, no. 6, pp. 707-712, Nov. 2000.

[13] M. L. Fowler, "Phase-based frequency estimation: A review," Digit Signal Process., vol. 12, no. 2, pp. 590-615, Oct. 2002.

[14] M. D. Macleod, "An improved fast estimator of the frequency of a single complex tone," in Proc. Sixth IMA Int. Conf. Math. Signal Process., Chicester, U.K., 2004, pp. 155-158.

[15] T. Brown and M. M. Wang, "An iterative algorithm for single-frequency estimation," IEEE Trans. Signal Process., vol. 50, no. 11, pp. 2671-2682, Nov. 2002.

[16] Z. Zhang, A. Jakobsson, M. D. Macleod, and J. A. Chambers, "Statistically and Computationally Efficient Frequency Estimation of a Single Tone,' Dept. Elect. Eng., Karlstad Univ., Karlstad, Sweden, Tech. Rep. EE-2005-02, 2005. 